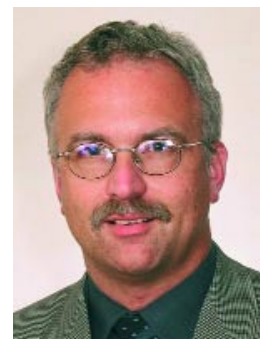

Ralf Gold

\section{Neue Therapieansätze bei der Multiplen Sklerose}

\author{
Ralf Gold ${ }^{1}$, Bernd C. Kieseier², Hans-Peter Hartung ${ }^{2}$ \\ ${ }^{1}$ Institut für MS-Forschung, Bereich Humanmedizin und Gemeinnützige Hertie-Stiftung, \\ Universität Göttingen \\ ${ }^{2}$ Neurologische Klinik und Poliklinik, Universität Düsseldorf
}

psychoneuro 2004; 30 (7): 375-379
$\mathrm{D}$ ie multiple Sklerose (MS) ist neben der Epilepsie die häufigste neurologische Erkrankung des jungen Erwachsenalters (20.-40. Lebensjahr) (27). Bei vielen dieser jungen Patienten führt die MS ohne spezifische Immuntherapie bereits nach wenigen Jahren zu einer signifikanten Behinderung mit Einschränkung der Gehstrecke auf unter $500 \mathrm{~m}$ (2). Auch die Diagnose einer sogenannten „benignen MS“ ist unserer Ansicht nach nur mit Einschränkungen anwendbar: dieser Terminus beschrieb ursprünglich Patienten, die nach 15 Jahren Krankheitsverlauf noch (evtl. mit Gehstock) selbst gehfähig waren. Dabei unberücksichtigt blieben kognitive Einschränkungen sowie die Tatsache, dass die meisten der jung Erkrankten mit dieser Behinderung oft erst das vierte Lebensjahrzehnt und damit nicht einmal die Hälfte der heutigen Lebenserwartung erreicht haben. Auf der Basis der von uns betreuten Patienten gehen wir vor diesem Hintergrund von weniger als $5 \%$ sog. benigner Verläufe aus.

Das morphologische Substrat dieser bleibenden Behinderung ist die Zerstörung von Myelinscheide und Axon, der sog. axonale Schaden, der schon zu Beginn des 20. Jahrhunderts beschrieben wurde und durch die Arbeit von Trapp (30) wie-

Die Pulstherapie mit Glukokortikosteroiden (GS) ist zur Zeit die einzige etablierte Behandlung einer akuten Verschlechterung bei MS, für die auch Studien relativ guten Evidenzgrades vorliegen. Bei Nonresponse mit schweren Ausfallssymptomen kann eine Plasmapherese-Serie erwogen werden. Zur immunmodulatorischen Basistherapie werden heute Interferone und Glatiramerazetat möglichst frühzeitig eingesetzt, um die Erfolgschancen der Langzeittherapie zu erhöhen. Bei hoher Schubfrequenz bzw. sekundär progredientem Verlauf ist Mitoxantron in Deutschland zugelassen. Neuartige Therapieansätze zur Behandlung des Schubs sowie zur Immunmodulation beeinflussen die fehlgeleitete Autoimmunreaktion auf verschiedenen Ebenen über die Induktionsphase der Immunantwort mit Antigenpräsentation und Kostimulation, die Adhäsion und Transmigration von Entzündungszellen und die lokale Zytotoxizität sowie Effektormechanismen. Vielversprechend sind u.a. der inhibierende VLA-4 Antikörper Natalizumab sowie neurotrophe Zytokine wie LIF (leucemia inhibitory factor).

der ins Zentrum des allgemeinen Interesses gerückt ist. Neben dieser körperlichen Einschränkung kommt es auch zu einer organisch-bedingten Minderung von Kognition und Ausdauerbelastbarkeit, deren Substrat in der neuropathologisch und kernspintomographisch belegten Hirnatrophie liegt $(6,16)$.

Es ist heute unbestritten, dass die MS eine heterogene Erkrankung ist. Basierend auf molekular-pathologischen Untersuchungen haben Lassmann und Mitarbeiter (19) versucht, eine Einteilung für die verschiedenen Verlaufsformen der MS zu definieren. Wenngleich die Patienten dieser Untersuchungen durch die in-vivo Hirnbiopsie selektioniert sind und daraus eine mögliche Verfälschung resultiert, konnten bestimmte Schädigungsmuster defi- niert werden, die z.B. mit Sonderformen wie der Neuromyelitis optica (Devic Erkrankung) (22), der Baloschen Sklerose oder auch primär progredienter MS korrelieren. Dies dient auch als Rationale für die Anwendung neuartiger Immuntherapien.

V.a. bei schubförmig-rezidivierenden (RR-MS), aber auch sekundär chronisch-progredienten Verläufen der MS (SP-MS) liegt nun eine Vielzahl von Therapiestudien hohen Evidenzgrades mit immunmodulatorischen und immunsuppressiven Substanzen vor, die 1999 die Grundlage für ein erstes Konsensuspapier der deutschsprachigen MS-Therapeuten bildeten (25). In den Folgejahren wurden diese Empfehlungen dem allgemeinen Fortschritt der Therapiestudien angepasst. Wir 
werden im folgenden neuartige Therapieansätze beschreiben, die bereits innerhalb kurzer Zeit zu deutlichen Fortschritten in der MS-Therapie führen können.

\section{Behandlung schubförmiger Verschlechterungen - Basistherapie und Eskalation}

Als „Schub“ werden nach Ausschluss physiologischer Schwankungen akute, ohne assoziierte Infekte oder Fieber auftretende neurologische Ausfälle bzw. eine Verschlechterung vorbestehender Symptome bezeichnet, die mindestens 24 Stunden lang anhalten (23). Symptome die innerhalb von vier Wochen nach der letzten schubförmigen Verschlechterung auftreten, werden immer noch zum selben „Schub“ gerechnet. Auch transiente Ausfälle durch Erhöhung der Körpertemperatur (z.B. Sauna, Sport; Uhthoff Phänomen) oder sog. Paroxysmen zählen nicht als Schub.

Die Schubtherapie mit Glukokortikosteroiden (GS) ist zur Zeit die einzige etablierte Behandlung einer akuten Verschlechterung bei MS, für die auch Studien relativ guten Evidenzgrades vorliegen (13). Die immunsuppressiven Wirkungen der Glukokortikosteroide erstrecken sich auf unterschiedliche humorale und zelluläre Immunprozesse. Die meisten dieser Effekte werden nach Stunden oder Tagen auf genomischer Ebene erzielt. Darüber hinaus weisen experimentelle Daten aus verschiedenen Modellen dosisabhängig rasche nicht-genomische Effekte nach (18), die via Induktion von TZellapoptose zu einem beschleunigtem Rückgang der Entzündung führen (9).
Auf empirischer Basis verwenden die meisten Untersuchungen Methylprednisolon für die sog. „Pulstherapie“ des MS-Schubes. Wir schlagen das in Tabelle 1 gezeigte Vorgehen vor. Falls auch nach wiederholter Pulstherapie schwere Ausfallssymptome wie Querschnittsmyelitis oder kompletter Visusverlust bestehen, kann mit dem Patienten die Möglichkeit einer Plasmapherese-Serie (Tab. 2) besprochen werden. Das pathogenetische Rational beruht darauf, dass bei solchen Patienten vorwiegend humoral vermittelte Läsionen die Krankheitssymptomatik verursachen, was aus histopathologischen Studien (19) prinzipiell gut belegt ist. Bei einer Sonderform der MS, der Neuromyelitis optica (Devic Erkrankung) sind solche Pathomechanismen ebenfalls eindeutig belegt (22).

Untersuchungen von Weinshenker et al. (33) zeigten in einer kontrollierten Studie mit Klasse-I-Evidenz eine bis zu 50\%ige Erfolgschance einer Plasmapheresetherapie, wobei ein Therapiebeginn innerhalb von vier bis sechs Wochen nach dem Schub die Erfolgschancen deutlich erhöht (17). Unsere eigenen Erfahrungen umfassen v.a. Patienten mit schwerer Optikusneuritis. Wir stellten bei unseren Patienten bei Durchführung von vier Plasmapheresen $(50 \mathrm{ml} / \mathrm{kg} \mathrm{KG}$ ) innerhalb von vier bis sechs Wochen nach Beginn der steroidresistenten Symptomatik eine Besserung um 60\% fest (28).

\section{Neuartige Therapieansätze zur Behandlung des Schubs sowie zur Immunmodulation}

Aus der Grundlagenforschung und experimentellen Modellen ist

\section{Tab.1 Durchführung einer Kortisonpulstherapie beim MS-Schub:}

- Laboruntersuchungen zum Infektausschluss, Rö-Thorax

- morgendliche i.v.-Gabe von 500-1000 mg Kortison (bevorzugt Methylprednisolon) über 3-5 Tage

- orale Therapie mit $1 \mathrm{mg} / \mathrm{kg}$ Methylprednisolon oder 1,25 mg/kg Prednisolon morgens bis Tag 14 anschließen, dann über 20-10-0-10 mg über vier Tage absetzen

- Beachten von gastrischen Nebenwirkungen, evtl. psychotropen Effekten der Glukokortikosteroide; deshalb Erstbehandlung immer unter stationären Bedingungen

- bei MS-Schüben und Optikusneuritis kann bei guter Remission nach i.v.-Phase auf orale Weitergabe verzichtet werden

- bei erneuter, sekundärer Verschlechterung nach Pulstherapie Wiederholung möglich modifiziert nach (11)

bekannt, dass eine fehlgeleitete Autoimmunreaktion auf verschiedenen Ebenen beeinflusst werden kann:

- die Induktionsphase der Immunantwort mit Antigenpräsentation und Kostimulation

- die Adhäsion und Transmigration von Entzündungszellen

- lokale Zytotoxizität und Effektormechanismen.

Basierend auf diesen Erkenntnissen wurden in den letzten Jahren eine Reihe von molekularen Therapiestudien der MS gestartet, die leider ergebnislos oder sogar unerwartet negativ ausfielen (34). Gerade hochselektive und in Modellen wirksame Ansätze wie Vakzination mit T-Zellrezeptoren, Neutralisation von TNF- $\alpha$ oder sog. mutierte Peptidliganden schlugen fehl. Die Zytokinnetzwerke bei MS werden demnach offensichtlich sehr differenziert reguliert.

Neueste experimentelle Studien legen nahe, dass durch den Einschluss von GS in lang zirkulierende Liposomen bereits durch Einmalgabe eine höhere Effizienz bei gleichzeitiger selektiver Anreicherung im entzündeten Gehirngewebe erreicht werden kann (29). Momentan sind bereits in der Rheumatologie klinische Phase-II-Studien in Gang, um die Übertragung dieser Paradigmen auf menschliche Erkrankungen zu überprüfen. Auch für die MS-Therapie könnte sich hier in naher Zukunft eine deutliche Verbesserung der Schubtherapie entwickeln.

Von den momentan in klinischen Studien untersuchten Substanzen besitzt der inhibierende VLA-4 Antikörper Antegren ${ }^{\circledR}$ (Natalizumab) aufgrund der sehr guten Ergebnisse der Phase-II-Studie (24) ein hohes Potential, nach Abschluss der beiden laufenden Zulassungstudien (AFFIRM und SENTINEL; Sponsor Biogenidec) ein festes Element in der Basistherapie der MS zu werden. Das Adhäsionsmolekül VLA-4 auf Leukozyten stellt ein essentielles Erkennungsmolekül für entzündliche Leukozyten dar, um die Barriere der Blut-Hirnschranke überwinden zu können. Daraus erklärt sich auch die 
extrem starke Reduktion der kernspintomographisch-belegten Gadolinium-Aufnahme in der Phase-IIStudie (24). Gleichzeitig wird dieser Mechanismus nicht in anderen Immunorganen verwendet, so dass sich keine generelle Schwäche der Infektabwehr entwickelt. In eigenen Untersuchungen konnten wir nach VLA-4 Blockade in experimentellen Modellen einen sehr raschen Rückgang der Entzündungsreaktion feststellen (20), so dass in Analogie zu einer früheren exploratorischen kleinen Studie (31) zukünftig auch eine Rolle als Schubtherapie diskutiert werden könnte. Des weiteren könnte die Art der Applikation von Antegren, als Kurzinfusion in vierwöchigen Intervallen, vielen MS-Patienten mit „Spritzenphobie“ und Angst vor häufiger Selbstinjektion entgegenkommen.

4

Immunmodulatorische Basistherapie mit Interferonen und Glatiramerazetat

$\mathrm{Zu}$ den verschiedenen Interferon- $\beta($ IFN- $\beta) 1 b$ - und IFN- $\beta 1 \alpha-$ Präparaten liegen mittlerweile breite Erfahrungen aus acht Jahren Zulassung und Anwendung bei der MS vor. Therapiespezifische Nebenwirkungen werden mittlerweile gut verstanden bzw. beherrscht (1). Auch ist die Langzeitgabe gefahrlos, und trotz gegenlautender einzelner Arbeiten (4) gehen die meisten Therapeuten aufgrund der erweiterten Verlaufsbeobachtungen von einem anhaltenden und relevanten Wirkeffekt aus. Wahrscheinlich entfalten IFN-Präparate ihre Hauptwirkung an der Blut-Hirnschranke (15). Nach heutigem Kenntnisstand erhöht ein frühzeitiger Einsatz von IFN-Präparaten die Erfolgschance der Langzeittherapie.

Mit Glatiramerazetat (GLAT) wurde erstmals eine antigenspezifische Therapie bei der MS erfolgreich eingesetzt. GLAT ist ein synthetisches Oligopeptid aus den vier Aminosäuren L-Glutaminsäure, L-Lysin und L-Alanin, L-Tyrosin in zufälliger Mischungsreihenfolge und Größe. Eigentlich bestand die Absicht mit der Entwicklung von GLAT in den 60er Jahren am Weizmann Institut in Rehovot, Israel, Peptide zu erhal- ten, die den immunbiologischen Eigenschaften von basischem Myelinprotein (MBP) als vermutetem Antigen ähnelten. Als „Zufallsbefund“ ergab sich, dass GLAT zwar nicht pathogen war, aber eine immuninhibitorische Wirkung hatte. In der zulassungsrelevanten Studie wurden täglich $20 \mathrm{mg}$ GLAT s.c. injiziert.

Obwohl die Wirkmechanismen von GLAT in den letzten Jahren intensiv untersucht wurden, ist der letztlich therapierelevante Effekt noch offen (10). Neuerdings ist $\mathrm{zu}$ den primär-immunologischen Mechanismen auch ein neurobiologischer Aspekt der GLAT-Wirkung hinzugekommen: GLAT-spezifische T- Zellen sezernierten nach Stimulation antigenspezifisch vermehrt BDNF (brain derived neurotrophic factor), ein Neurotrophin das potentielle Relevanz für das Überleben von Gliazellen und Axonen in der entzündlichen Läsion haben könnte (35). Zur Zeit werden Befunde aus der kernspintomographischen Zulassungsstudie damit in Zusammenhang gebracht: ergänzend zur Beeinflussung aktiver Entzündungsherde konnte eine Reduktion der Gewebeschädigung unter GLAT durch seltenere Umwandlung neuer Läsionen in sog. „Black Holes" nachgewiesen werden $(4,5)$. Für die schlüssige Beurteilung der funktionellen Relevanz von BDNF Produktion durch GLAT werden allerdings weitere experimentelle Studien benötigt.

\section{Eskalierende Immuntherapie mit Mitoxantron}

Mitoxantron (Ralenova ${ }^{\circledR}$ ), ein vollsynthetisch hergestelltes Aminoanthracenedion, ist ein Zytostatikum aus der Klasse der Anthracenedione und den Anthrazyklinen Doxorubicin und Daunorubicin verwandt. In der Neurologie wurde Mitoxantron erstmals von Gonsette 1987 bei MS Patienten verwendet, ausgehend von der sehr guten Wirkung dieser Substanz in Tiermodellen der multiplen Sklerose. Im Gegensatz zu vielen anderen Therapeutika erwies sich Mitoxantron auch bei der menschlichen Erkrankung als hochwirksam und insbesondere dem Cyclophosphamid überlegen (12).

\section{Tab. 2 Eskalierende Schubtherapie mit Plasmapherese}

- schwere Ausfallssymptome (Ataxie, Visusverlust, Querschnitt)

- wiederholte Kortisonpulstherapie (typischerweise jeweils 1000 mg über 3-5 Tage) erfolglos

- Beginn spätestens innerhalb von sechs Wochen nach Schub

- bisher keine gesicherte Datenlage für den alternativen Einsatz von intravenösen 7S-Immunglobulinpräparaten

Mitoxantron interkaliert wie Doxorubicin in die DNA, und bewirkt über eine Interaktion mit der Topoisomerase-2 Einzel- und Doppelstrangbrüche. Verschiedene in-vitroStudien deuten darauf hin, dass der zytotoxische Effekt von Mitoxantron bei chronischen Leukämien über die Induktion von Apoptose vermittelt wird; auch in Tiermodellen der MS führt Mitoxantron zu vermehrter TZellapoptose $(26,32)$. Man muss allerdings an dieser Stelle anführen, dass vergleichbare Wirkungen auch bei anderen Zytostatika wie dem Cyclophosphamid gefunden wurden und sich der überlegene therapeutische Effekt von Mitoxantron wahrscheinlich nicht alleine daraus erklärt.

Wir empfehlen die Ersttherapie mit Mitoxantron (Ralenova ${ }^{\circledR}$ ) unter stationären Bedingungen, wo mit suffizienter antiemetischer Behandlung im allgemeinen eine sehr gute Verträglichkeit beobachtet wird. Bereits schwerer betroffene MS-Patienten berichten mitunter über mehrtägige Abgeschlagenheit nach den Infusionen. Wegen der potentiell kardiotoxischen Wirkung von Mitoxantron muss vor Therapiebeginn durch EKG und Echokardiographie eine kardiale Vorschädigung ausgeschlossen werden, und auch zumindest alle zwölf Monate unter Therapie eine kardiologische Kontrolluntersuchung erfolgen. Wegen der potentiellen Kardiotoxizität wird in der Neurologie eine kumulative Lebenszeitdosis von $140 \mathrm{mg} / \mathrm{m}^{2}$ Körperoberfläche als Grenzdosis angesehen. Durch die renale Elimination von Mitoxantron tritt am Tag nach der Infusion eine harmlose Blaugrünverfärbung des Urins auf. 
Oft wird auch ein leichtgradiger Haarausfall ca. vier Wochen nach Erstinfusion berichtet, wobei wir an unseren Patienten ( $n>400$ seit Anfang der 90er Jahre) nie eine relevante Alopezie beobachtet haben. Zyklusstörungen oder sogar mehrmonatige Amenorrhö können bei bis zu 50\% der behandelten Frauen auftreten. Trotzdem muss eine suffiziente Kontrazeption erfolgen.

Als Zytostatikum kann Mitoxantron theoretisch analog zu Azathioprin oder Cyclophosphamid die Entwicklung von Sekundärmalignomen begünstigen; aus der Weltliteratur sind hierzu nur wenige Fälle einer akuten Leukämie bekannt (7). Allerdings sollte über diese schwere Nebenwirkung eine dokumentierte Aufklärung erfolgen.

Nach verschiedenen kleineren, positiv verlaufenden Studien mit Mitoxantron bei Patienten mit hochaktiver, schubförmiger MS führten die Ergebnisse der Europäischen Dosisvergleichstudie MIMS (14) zur Zulassung von Mitoxantron bei hoher Schubfrequenz bzw. sekundär progredientem Verlauf in der Dosierung von $12 \mathrm{mg} / \mathrm{m}^{2}$ Körperoberfläche Mitoxantron. Ein dosisabhängiger, signifikanter Effekt von Mitoxantron auf Schubrate, Krankheitsprogression und kernspintomographische Aktivität bei beiden Verlaufsformen der Erkrankung (schubförmig und sekundär progredient) konnte hierbei nachgewiesen werden. Aufgrund dieser Daten ist Mitoxantron im Herbst 2000 in den USA von der FDA (Food and Drug Administration, amerikanische Arzneimittelzulassungsbehörde) für die Therapie rasch-progredienter MS-Verläufe unter dem Handelsnamen Ralenova ${ }^{\circledR}$ zugelassen worden und im Dezember 2002 endlich auch in Deutschland. Nach der Erstinfusion unter stationären Bedingungen (s.o.) kann im allgemeinen die Weiterbehandlung in dreimonatlichen Abständen auf ambulanter Basis erfolgen. Bei besonders hoher Krankheitsaktivität kann im Einzelfall in Absprache mit erfahrenen Zentren auch initial eine Induktionstherapie mit drei Infusionen in monatlichen Abständen durchgeführt werden.
Mit der Zulassung von Ralenova ${ }^{\circledR}$ ist dies nun offiziell verordnungsfähig. Eine weitere klinische Studie zur Wirksamkeit niedrigerer Dosierungen ist momentan angelaufen. Der Einsatz von Mitoxantron sollte in Anbetracht der vorliegenden Studien nach sorgfältiger Abwägung der Indikation in erster Linie bei Patienten mit hochaktivem (>2 Schübe/Jahr) schubförmigen Verlauf und progredientem bzw. progredient-schubförmigem Verlauf (Progression > 1 EDSS Punkt/Jahr) erwogen werden.

\section{Zukünftige Therapie- strategien}

Ein bisher nur in experimentellen und retrospektiven Studien geprüfter Ansatz beschäftigt sich mit der Rolle von neurotrophen Zytokinen als Überlebensfaktoren für Oligodendroglia, und damit letztlich Vermittler von Demyelinisierung mit nachfolgendem axonalen Schaden. Sowohl das CNTF (ciliärer neurotropher Faktor) als auch LIF (leucemia inhibitory factor) spielen hier eine Schlüsselrolle: fehlt das CNTF, tritt im Mausmodell für die MS vermehrte Demyelinisierung, apoptotischer Tod von Oligodendrozyten und letztlich vermehrter axonaler Schaden auf (21). Parallel zu unseren Untersuchungen konnte eine australische Arbeitsgruppe zeigen, dass durch die systemische Gabe von LIF Oligodendrozyten vor Zelltod geschützt wurden und somit die Erkrankung milder verlief (3). Diese experimentelle Studien wurden durch eine retrospektive Analyse eines MS-Patientenkollektivs unterstützt, in der Patienten mit einem Defekt beider Allele des CNTF-Gens früher und schwerer an MS erkrankten (8).

Somit besteht die Möglichkeit, dass zukünftig neben immunologischen Studien auch neurobiologische Untersuchungen bei MS an Bedeutung gewinnen.

\section{Schlussfolgerung}

Parallel zu Fortschritten in der Pathogeneseforschung kam es im letzten Jahrzehnt zu einer deutlichen Verbesserung der Immuntherapie für Patienten mit schubförmiger oder sekundär progredienter MS. Die ersten Daten zu der verbes- serten Langzeitprognose der Erkrankung unter Immuntherapie lassen nicht nur eine Besserung der Behinderung, sondern auch der Kognition und Ausdauerbelastbarkeit erwarten. Für primär chronisch-progrediente MS sind leider vergleichbare Fortschritte noch nicht realisiert worden.

Corticosteroid-pulse therapy is still the mainstay for the treatment of acute relapses of MS. In patients who fail to respond to steroid pulse therapy, plasmapheresis may lead to rapid and sustained improvement. Immunomodulatory treatment with interferon or glatirameracetate serves as basis for longterm therapy in relapsing-remitting MS. In patients with high relapse-rate or secondary disease progression mitoxantrone can be used in escalating treatment regimens. Novel immunotherapies act at different levels: they target the induction phase with antigen presentation and costimulation, adhesion and transmigration of inflammatory cells and local cytotoxicity. Promising agents include anti-VLA-4 antibodies (natalizumab TM), and neurotrophic cytokines such as LIF (leucemia inhibitory factor).

\section{Key Words}

multiple sclerosis - glucocorticosteroid therapy - plasma exchange - mitoxantrone - Natalizumab

\section{Literatur}

1. Bayas A, Rieckmann P. Managing the adverse effects of interferon-beta therapy in multiple sclerosis. Drug Saf 2000; 22: 149-159

2. Brex PA, Ciccarelli O, O'Riordan Jl, Sailer $\mathrm{M}$, Thompson AJ, Miller DH. A longitudinal study of abnormalities on MRI and disability from multiple sclerosis. New England Journal of Medicine 2002; 346: 158-164

3. Butzkueven H, Zhang JG, Hanninen MS et al. LIF receptor signaling limits immunemediated demyelination by enhancing oligodendrocyte survival. Nature Medicine 2002; 8: 613-619

4. Filippi M, Rovaris M, Rocca MA, Sormani MP, Wolinsky JS, Comi G. Glatiramer acetate reduces the proportion of new MS lesions evolving into „black holes“. Neurology 2001; 57: 731-733

5. Filippini G, Munari L, Incorvaia B et al. Interferons in relapsing remitting multiple sclerosis: a systematic review. Lancet 2003; 361: 545-552

6. Fisher E, Rudick RA, Cutter $G$ et al. Rela- 
tionship between brain atrophy and disability: an 8-year follow-up study of multiple sclerosis patients. Multiple Sclerosis 2000; 6: 373-377

7. Ghalie RG, Mauch E, Edan G et al. A study of therapy-related acute leukaemia after mitoxantrone therapy for multiple sclerosis. Multiple Sclerosis 2002; 8: 441-445

8. Giess R, Maurer M, Linker R et al. Association of a null mutation in the CNTF gene with early onset of multiple sclerosis. Archives of Neurology 2002; 59: 407-409

9. Gold R, Buttgereit F, Toyka KV. Mechanism of action of glucocorticosteroid hormones: possible implications for therapy of neuroimmunological disorders. Journal of Neuroimmunology 2001; 117: 1-8

10. Gold R, Heidenreich F, Kappos L. Immunotherapy of multiple sclerosis with glatiramer acetate mechanisms of action and results from therapeutic trials. Aktuelle Neurologie 2002; 29, 345-351

11. Gold R, Toyka KV. Immuntherapie neurologischer Erkrankungen. Bremen, UNI-MED Verlag, 2001

12. Gonsette RE, Demonty L. Mitoxantrone: a new immunosuppressive agent in multiple sclerosis. In: Recent advances in multiple sclerosis therapy. Gonsette RE, Delmotte P (eds). Elsevier Science Publishers 1989; 161-164

13. Grauer O, Offenhausser M, Schmidt J, Toyka KV, Gold R. Glucocorticosteroid therapy in optic neuritis and multiple sclerosis: evidence from clinical studies and practical recommendations. Nervenarzt 2001; 72, 577-589

14. Hartung HP, Gonsette R, Konig N et al. Mitoxantrone in progressive multiple sclerosis: a placebo-controlled, doubleblind, randomised, multicentre trial. Lancet 2002; 360, 2018-2025

15. Hartung HP, Kieseier BC. Targets for the therapeutic action of interferon-beta in multiple sclerosis [editorial]. Ann Neurol 1996; 40, 825-826

16. Kalkers NF, Ameziane N, Bot JCJ, Minneboo A, Polman CH, Barkhof F. Longitudinal brain volume measurement in multiple sclerosis - Rate of brain atrophy is independent of the disease subtype. Archives of Neurology 2002; 59, 1572-1576

17. Keegan M, Pineda AA, McClelland RL, Darby $\mathrm{CH}$, Rodriguez $\mathrm{M}$, Weinshenker BG. Plasma exchange for severe attacks of CNS demyelination: Predictors of response. Neurology 2002; 58, 143-146

18. Krauss S, Brand MD, Buttgereit F. Signaling takes a breath - new quantitative perspectives on bioenergetics and signal transduction. Immunity $2001 ; 15$, 497-502 19. Lassmann H, Bruck W, Lucchinetti C. Heterogeneity of multiple sclerosis pathogenesis: implications for diagnosis and therapy. Trends in Molecular Medicine 2001; 7, 115-121

20. Leussink VI, ZettI UK, Jander S et al. Blockade of signaling via the very late antigen VLA-4; and its counterligand vascular cell adhesion molecule-1 VCAM-1; causes increased $\mathrm{T}$ cell apoptosis in experimental autoimmune neuritis. Acta Neuropatholo- gica 2002; 103, 131-136

21. Linker RA, Maurer M, Gaupp $S$ et al. CNTF is a major protective factor in demyelinating CNS disease: A neurotrophic cytokine as modulator in neuroinflammation. Nature Medicine 2002; 8, 620-624

22. Lucchinetti CF, Mandler RN, McGavern $D$ et al. A role for humoral mechanisms in the pathogenesis of Devic's neuromyelitis optica. Brain 2002; 125, 1450-1461

23. McDonald WI, Compston A, Edan G et al. Recommended diagnostic criteria for multiple sclerosis: Guidelines from the International Panel on the Diagnosis of Multiple Sclerosis. Annals of Neurology 2001; 50 , 121-127

24. Miller DH, Khan OA, Sheremata WA et al. A controlled trial of natalizumab for relapsing multiple sclerosis. New England Journal of Medicine 2003; 348, 15-23

25. MS-Therapie Konsensusgruppe MSTKG. Immunmodulatorische Stufentherapie der Multiplen Sklerose. Nervenarzt 1999; 70 , 371-386

26. Neuhaus et al. Zur Publikation eingereicht

27. Noseworthy JH, Lucchinetti C, Rodriguez M, Weinshenker BG. Multiple sclerosis. New England Journal of Medicine 2000; 343 , 938-952

28. Ruprecht et al. Neurology 2004; im Druck

29. Schmidt J, Metselaar JM, Wauben MH, Toyka KV, Storm G, Gold R. Drug targeting by long-circulating liposomal glucocorticosteroids increases therapeutic efficacy in a model of multiple sclerosis. Brain 2003; 126, 1895-1904

30. Trapp BD, Peterson J, Ransohoff RM, Rudick R, Mörk S, Bö L. Axonal transection in the lesions of multiple sclerosis. New England Journal of Medicine 1998; 338, 278-285

31. Tubridy N, Behan PO, Capildeo R et al. The effect of anti-alpha4 integrin antibody on brain lesion activity in MS. The UK Antegren Study Group [see comments]. Neurology 1999; 53, 466-472

32. Weilbach et al. Zur Publikation eingereicht

33. Weinshenker BG, O'Brien PC, Petterson $\mathrm{TM}$ et al. A randomized trial of plasma exchange in acute central nervous system inflammatory demyelinating disease. Ann Neurol 1999; 46, 878-886

34. Wiendl H, Hohlfeld R. Therapeutic approaches in multiple sclerosis - Lessons from failed and interrupted treatment trials. Biodrugs 2002; 16, 183-200

35. Ziemssen T, Kumpfel T, Klinkert WEF, Neuhaus O, Hohlfeld R. Glatiramer acetatespecific T-helper 1-and 2-type cell lines produce BDNF: implications for multiple sclerosis therapy. Brain 2002; 125, 2381-2391

\section{Korrespondenzadresse:}

Prof. Dr. Ralf Gold

Institut für MS-Forschung

Waldweg 33

37073 Göttingen

E-Mail: r.gold@med.uni-goettingen.de

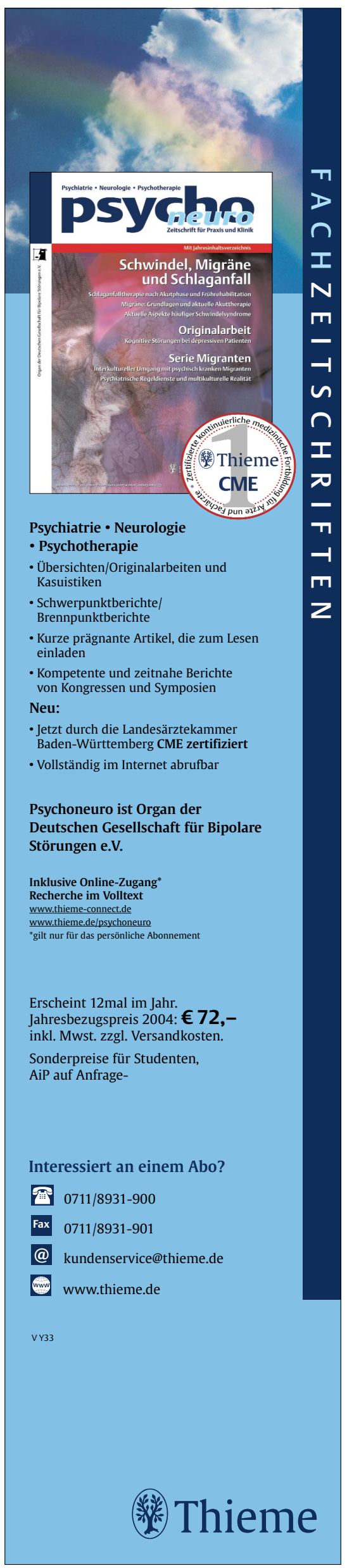

